\title{
A Basic Study on Sound Control System for Ultra-Compact Electric Vehicle by Using Masking
}

\author{
Taro Kato ${ }^{1}$, Hiroya Nakayama ${ }^{2}$, Hideaki Kato ${ }^{3, *}$ (i) and Takayoshi Narita ${ }^{3}$ (i) \\ 1 Course of Science and Technology, Tokai University, Kitakaname 4-4-1, Hiratsuka-shi, \\ Kanagawa 259-1292, Japan; 9btad004@mail.u-tokai.ac.jp \\ 2 Course of Mechanical Engineering, Tokai University, Kitakaname 4-4-1 Hiratsuka-shi, \\ Kanagawa 259-1292, Japan; 0cemm061@mail.u-tokai.ac.jp \\ 3 Department of Prime Mover Engineering, Tokai University, Kitakaname 4-4-1 Hiratsuka-shi, \\ Kanagawa 259-1292, Japan; norita@tsc.u-tokai.ac.jp \\ * Correspondence: hkato@tokai-u.jp; Tel.: +81-463-58-1211
}

Received: 19 March 2020; Accepted: 11 May 2020; Published: 15 May 2020

\begin{abstract}
In this study, we conducted a quantitative evaluation of the comfort of the interior of an electric vehicle (EV) using the brain wave appearance rate, which is part of the human biologic information that the initial stage of the proposed active noise control (ANC) system for ultra-compact EVs reveals. EVs have become easy-to-use mobility solutions and have been researched and actively developed focusing on using music characteristics. We performed fundamental testing of music including $1 / f$ fluctuation for the evaluation of ride comfort based on the relationship between the participant's heart rate and tempo of music using a driving simulator. The results suggest that if a passenger listened to music including a $1 / f$ fluctuation, then he/she could relax. Thus, it was concluded that if we could pre-grasp the passenger's biologic information of the heart rate and beats per minute for masking, then the comfort in the interior space could be improved even when using a driving simulator.
\end{abstract}

Keywords: active noise control; masking; brain wave; $1 / f$ fluctuation; tempo of music

\section{Introduction}

Ultra-compact electric vehicles (EVs) with a short width and wheelbases, which can accommodate one or two seats, are becoming increasingly popular. These vehicles can pass through narrow streets and are expected to be a new mode of personal transport in the city [1]. In addition, because the vehicle uses an electric motor as a power source instead of a gasoline engine or a diesel engine, it not only has a low environmental impact, but also does not generate noise from the engine. Therefore, the noise input into the vehicle mainly consists of a road noise from the tires and a wind noise. This noise causes a problem by deteriorating the comfort in the cabin [2-4]. A conventional vehicle usually installs a sound absorbing material in the cabin or a speaker for active noise control (ANC) [5]. However, due to the compactness of the ultra-compact EVs, installation of these devices is difficult. This causes the deterioration of the comfort of the vehicle. Therefore, we focused on a giant magnetostrictive actuator (GMA), which is small in size and generates a high output and proposed an ANC system using the wall vibration generated by the GMA, thereby confirming the silencing effect [6,7]. Furthermore, our studies are not only aimed at "noise reduction", but also at "pleasant sound" to control a comfortable space for several drivers.

However, research on the determination of the ride comfort of ultra-compact EVs has been predominantly conducted through questionnaires and sensorial evaluation [8]. Sensuous and subjective evaluation can measure human sensations directly. On the contrary, it is difficult to evaluate 
quantitatively these results, since these evaluations are self-report which depends on subjectivity and individual preferences of an experimental participant [9]. That is enough scientific and engineering research on human sensitivity have not been performed. In another research field, a masking that adds background music (BGM) to noised space has been proposed to reduce subjectively the noise of a person in the space. According to Haiyan Shu et al. [10], the discomfort caused by the noise in urban areas was decreased by masking the noise with music and water sounds. Furthermore, Nishiura reported a study on the subjective evaluation of comfortability attained using some masking sounds for noise [11]. The authors created a system that provided comfortable interior noise environment for ultra-compact EV users. We focused on the masking that uses sounds such as music to control the road and wind noise in the cabin. Even though there have been studies related to noise reduction in the cabins of ultra-compact EV by using the masking, none of them provides a quantitative evaluation based on biologic information.

In this study, we used a music to mask the interior noise in ultra-compact EVs and to improve the driver comfortability. For providing the driver comfort, we focused on the $1 / f$ fluctuation in the music [12] and the relationship between the heart rate and tempo of music [13]. Furthermore, it has not been researched about the usefulness of masking in the driving condition even though a driver uses masking during operating steering wheel in actual situation. We used electroencephalography (EEG) for obtaining the biologic information to quantitatively evaluate the comfort attained by masking the interior noise.

\section{Consideration on Comfortability by Using Biologic Information}

\subsection{Comfortability Evaluation by Brain Wave}

Several studies on the evaluation of comfort for interior spaces and rides adopt quantitative evaluation of a human's biologic information [14-16]. The brain wave which is one of biologic information is an electrical signal generated by brain accompanied by nerve call activity.

We diagnosed the dependence of the human's mental condition on the mental state, sensory stimulation and awareness level. In this experiment, we measured the brain wave under different sound conditions for quantitative evaluation using a brain wave meter (Alphatec IV supported by EEG Research Laboratory Corporation), as illustrated in Figure 1 [17,18]. This device measured brain wave and transformed to spectrum in each one second. The translated spectrum can be categorized to five bands of $\theta$ wave (4-6 Hz), Sawave (7-8 Hz), M $\alpha$ wave (9-11 Hz), F $\alpha$ wave $(12-14 \mathrm{~Hz})$ and $\beta$ wave $(15-23 \mathrm{~Hz})$. Some researchers investigated $\alpha$ wave which includes $S \alpha, M \alpha$ and $F \alpha$ wave by index of comfort evaluation and reported that the $\alpha$ wave appears when a human feel relaxed [19]. In this study, we considered $F \alpha$ wave, $M \alpha$ wave and $S \alpha$ wave as a single $\alpha$ wave.

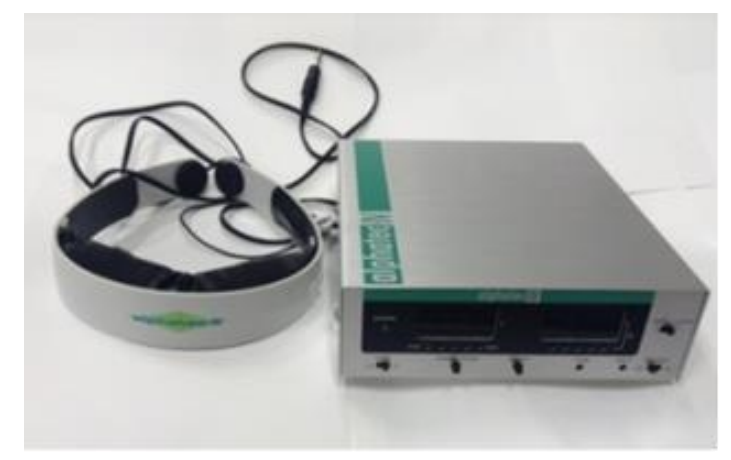

Figure 1. Brain wave meter.

Participants of this study wore the brain wave meter. Figure 2 shows a sample of the brain wave spectrum that was obtained for one second. From the figure, it can be seen that the frequency which has a maximum voltage in the brain wave spectrum was $20 \mathrm{~Hz}$ that belongs to $\beta$ wave. Therefore, 
we judged that $\beta$ wave most appeared in the second and counted the number of appearances of each brain wave in measurement time. We used $\alpha$ wave appearance rate for the evaluation index, which is defined as the ratio of $\alpha$ wave to all appearance brain wave in order to composition ratio in each frequency band within measurement time. Table 1 shows a sample of the appearance number of the maximum voltage in each brain wave group. From the measured brain wave, we calculated the $\alpha$ wave appearance rate by using the count of max voltage in each frequency for one minute. The $\alpha$ wave appearance rate is the percentage of $\alpha$ wave per whole brain wave as shown in the following equation:

$$
\mathrm{A}(\%)=\frac{S \alpha+M \alpha+F \alpha}{\theta+S \alpha+M \alpha+F \alpha+\beta} \times 100
$$

The comfortable evaluation criteria was based on the average of $\alpha$ wave appearance rate for one minute.

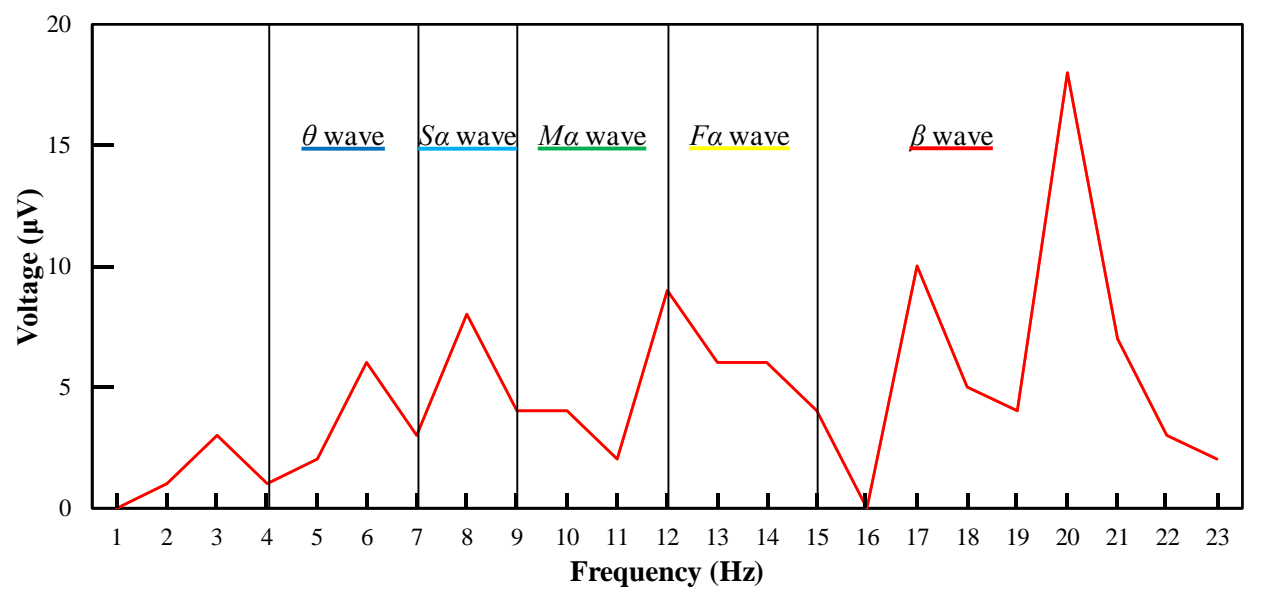

Figure 2. Sample result of measured brain wave of one second.

Table 1. Sample result of the total number in one minute of brain waves that appeared most strongly in the spectrum calculated every second.

\begin{tabular}{lccccc}
\hline Divide 5 type along to frequency & $\theta$ & $S \alpha$ & $M \alpha$ & $F \alpha$ & $\beta$ \\
\hline Count of max voltage each frequency & 8 & 13 & 11 & 9 & 19 \\
\hline
\end{tabular}

\subsection{Masking Technology}

The study on the improvement of comfort with respect to the noise by using the masking was gaining attention in various fields. Masking is one of the hearing phenomena. Signal A is difficult to hear if signal B exists for signal A because of the increase in the minimum audible range. The masking has difficulty in achieving complete masking when signal A can only be heard with difficulty.

Noise reduction in the vehicle interiors is extremely important for a driver to drive comfortably. There were studies related to the use of BGM as a masking technique for noise reduction in interior spaces. However, for the purpose of determining the driver comfort in ultra-compact EVs, studies mainly focus on questionnaires and organoleptic evaluations. In such cases, it has not been investigated enough scientific and engineering research on human sensitivity.

We proposed an ANC system for ultra-compact EVs as shown in Figure 3. Furthermore, we studied the interior sound control based on biologic information of driver. We aimed to conduct a quantitative evaluation for ride comfort to ensure that a driver feels relaxed and concentrated while driving $[6,7,20,21]$. 


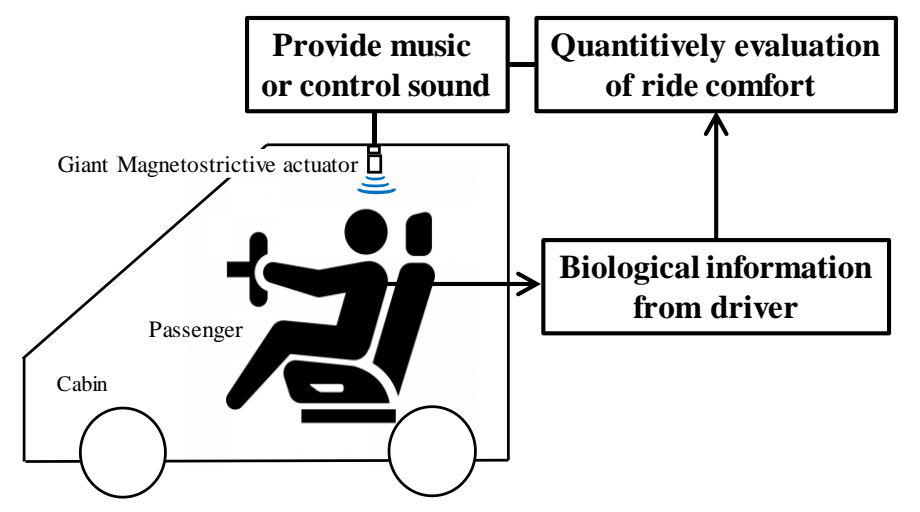

Figure 3. Active noise control (ANC) system for ultra-compact electric vehicle (EV) using giant magnetostrictive actuator (GMA) in future.

\section{Fundamental Consideration of $1 / f$ Fluctuation Including Music for Evaluation of Ride Comfort}

\subsection{Fundamental Consideration of Masking Music Including 1/f Fluctuation}

A $1 / f$ fluctuation has a distinct mathematical feature the power spectral density is proportional to the inverse of the Fourier frequency $f$ [22]. The $1 / f$ fluctuations in a natural phenomenon are included in the sunbeam shining through the branches of trees and shaking fire of a candle, etc.

Doi et al. investigated the effect of interior lighting control on the comfort of humans [23]. They focused on the fluctuation of flame light of a candle. They measured the lighting intensity of the candle and obtained the light power spectrum by Fourier Transform. Furthermore, they calculated the $1 / f$ fluctuation in the light by the change in emission intensity and power spectrum. According to the analyzed results, they controlled the interior lighting and evaluated the comfortability using biologic information of the participant. From the results obtained, they could substantiate the improvement in the participant's comfortability using lighting control.

We calculated power spectrum of music using for masking and analyzed $1 / f$ fluctuation. Regarding sound, the average of a regular sound and random sound can provide a relaxation effect. In general, various classic and modern music include a $1 / f$ fluctuation.

\subsection{Analysis of the $1 / f$ Fluctuation of the Music}

In experiments using masking, we selected music that would make the drivers feel more comfortable. We conducted a spectral analysis for the selected music pieces from start to end, to analyze the $1 / f$ fluctuation. The spectrum exhibited the discrete Fourier transform, as defined in the following equation:

$$
X(k \Delta f)=\frac{N}{T_{0}} \sum_{n=0}^{N-1} x(n \Delta t) e^{-j \frac{2 \pi}{N} k n}
$$

Here, $T_{0}$ is the music running time [s ( $\mathrm{s}=$ seconds)], $\Delta t$ is the sampling time $[\mathrm{s}], N$ is the sampling number of wav data, $x$ is the sampled music data, $\Delta f$ is the frequency resolution and $X$ is the amplitude of each frequency. In this experiment, for all cases, the sampling frequency of the music was $44.1 \mathrm{kHz}$ and music duration were three minutes.

Figure 4 shows a sample result of the spectral analysis of pink noise as obtained by the above equation. The figure shows the spectrum of sound data amplitude on the vertical axis and frequency on the horizontal axis. The fluctuation coefficient, $\lambda$ was obtained by the calculation of the spectral slope from $0.05 \mathrm{~Hz}$ to $0.5 \mathrm{~Hz}$. It reveals that the music piece whose coefficient was approximately-1 contained a $1 / f$ fluctuation. 


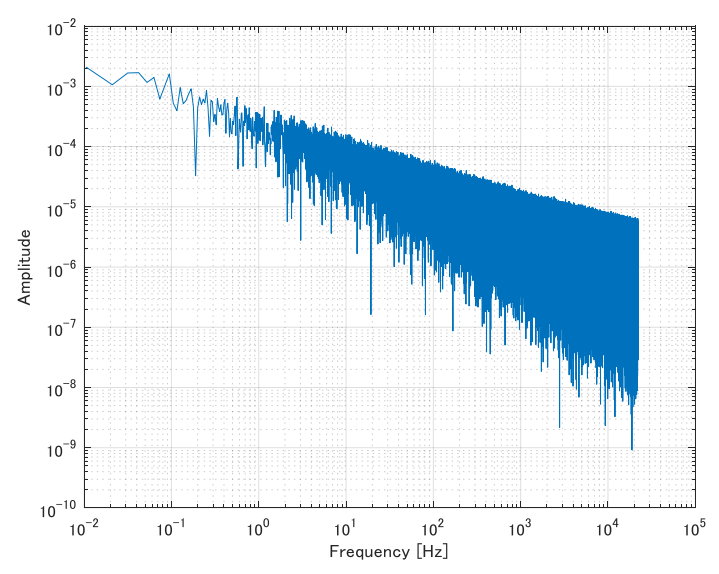

Figure 4. Sample of $1 / f$ fluctuation analysis.

In this study, we collected compact discs (CDs) of 1527 music pieces and converted them to wav files to analyze the $1 / f$ fluctuation. All the 1527 music pieces contained songs of either male or female singers. We analyzed the fluctuation coefficient $\lambda$, which was obtained by the fluctuation analysis of the wav file. From the results of the analysis, the 1527 music pieces that were used for masking and defined 340 music pieces whose fluctuation coefficients were in the range of -1 to -2 , indicating that they contained a $1 / f$ fluctuation.

\subsection{Experiment of the Relaxation Effect by the Music Including a 1/f Fluctuation}

We conducted an experiment to determine the relaxation effect on a participant by using 340 selected music pieces, which were analyzed by the method described in the previous sub-section. In this experiment, we conducted the quantitative evaluation of the comfort by measuring the brain wave when outputted road noise masked with the music during driving simulator.

Figure 5 depicts the participant attached to an electroencephalograph and sitting at rest in front of the driving simulator and speakers which output assumed road noise and music for masking. As an initial study, the use of music for masking interior noise was investigated by observing the relationship between the masking of interior noise and driver comfort. Driver comfort was evaluated using the biologic information obtained from the participant while he/she was using a simple driving simulator that could simulate real driving conditions. The participant's brain wave was measured while he/she listened to three music pieces for $3 \mathrm{~min}$ each. The three sound conditions considered in these experiments were (1) road noise, (2) road noise + music excluding $1 / f$ fluctuation, (3) Road noise + music including $1 / f$ fluctuation.

Before starting the measurements, the participant practiced on the simple driving simulator for $2 \mathrm{~min}$. After the practice, the participant's brain wave was measured for $3 \mathrm{~min}$, while he/she listened to the road noise music pieces and simultaneously operated the simulator. After the first brain wave measurement, the participant took a break for one minute while sitting on the chair. After the break, the participant was asked to choose his/her favorite masking music piece from the 1527 music pieces. For the next experiment, the participant's brain wave was measured for $3 \mathrm{~min}$, while he/she listened to the road noise + music excluding $1 / f$ fluctuation and simultaneously operated the simulator. After the experiment, the participant took a break as before. After the break, the participant was asked to choose a masking music piece among the 340 music pieces, which were confirmed to contain $1 / f$ fluctuation from the previous analysis. Finally, the participant's brain wave was measured for $3 \mathrm{~min}$, while he/she listened to road noise + music including $1 / f$ fluctuation and simultaneously operated the simulator. The average sound pressure level was $70 \mathrm{~dB}$, which was measured by the noise meter located at near participant's ear position. The experimental flow is shown in Figure 6. 


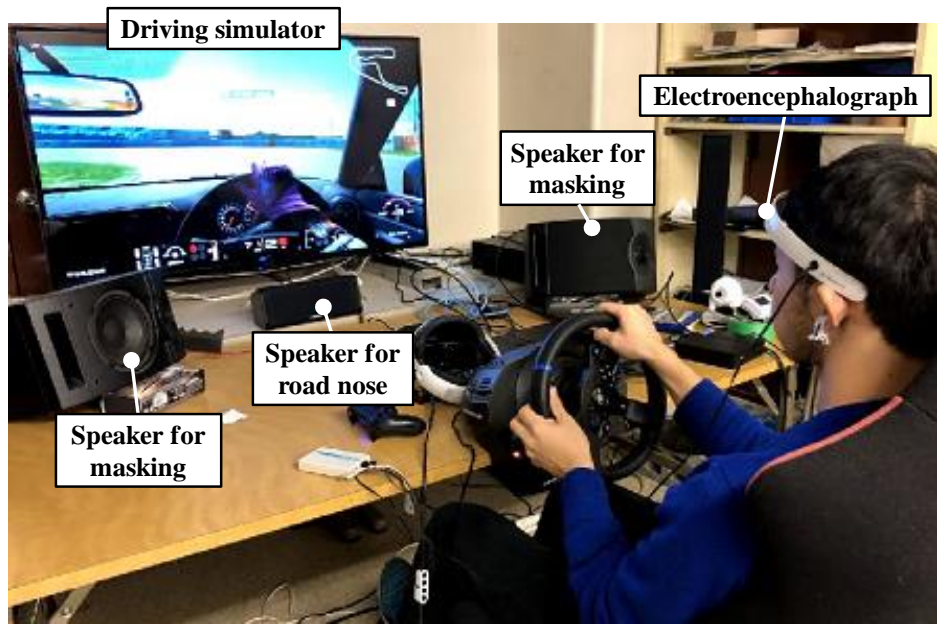

Figure 5. Experimental situation by using driving simulator.

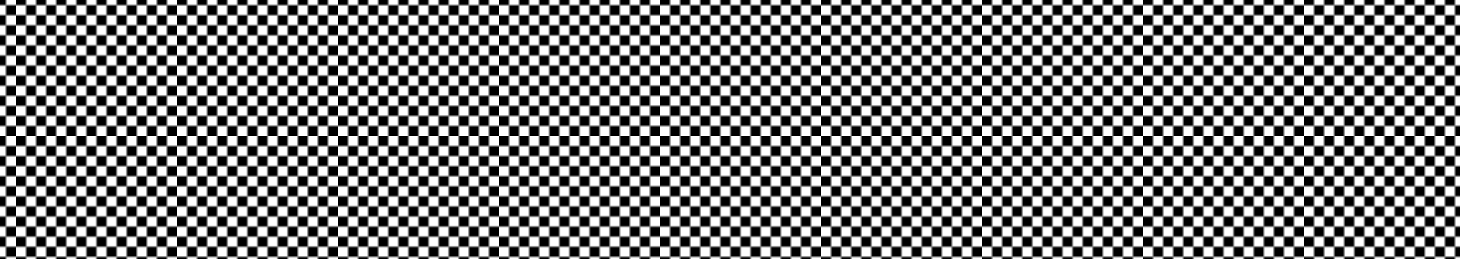

Figure 6. The experimental flow about the relaxation effect by the music including a $1 / f$ fluctuation.

In this experiment, there were 6 male participants who were university and graduate of university students. The participants were aged between 21 and 24 years. All the participants had a driving license and more than 3 years of driving experience. This experiment was approved by the Ethics Committee of Tokai University's "Research targeting humans". (The ethics committee approval code is 19058.)

\subsection{Experiment of the Relaxation Effect by the Music Including a 1/f Fluctuation}

Figure 7 depicts the experimental results for 6 participants. According to the experimental results, it was revealed that the $\alpha$ wave appearance rate for all participants increased when the road noise was masked by music including $1 / f$ fluctuation in comparison with when the road noise was masked by music excluding $1 / f$ fluctuation. Therefore, we can conclude that masking music including $1 / f$ fluctuation must be used to create a comfortable space in the cabin.

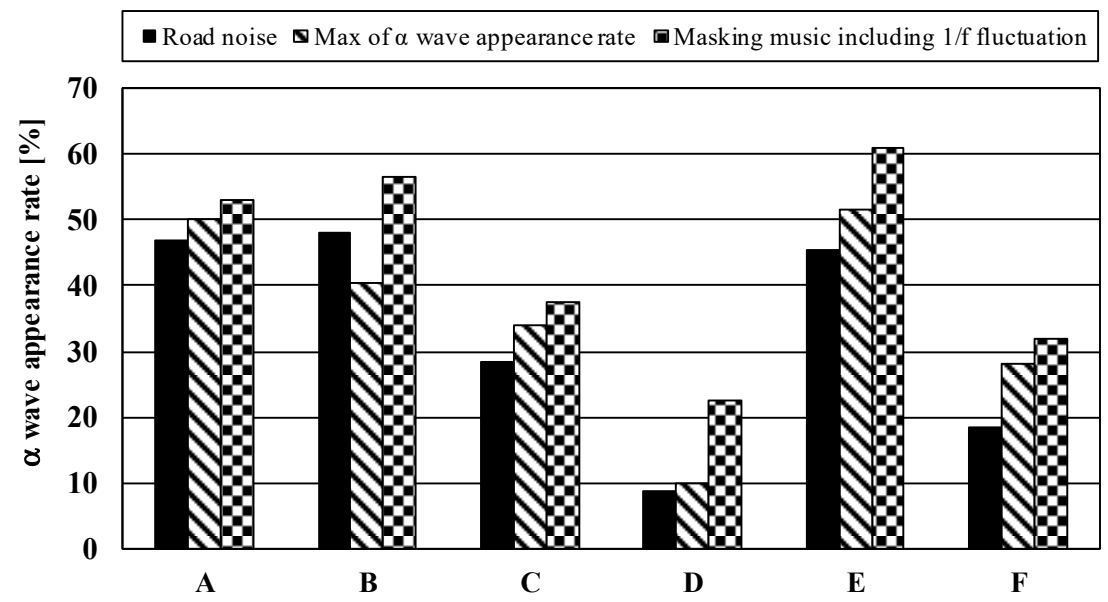

Figure 7. Experiment result of the rate of participants for each music. 


\section{Fundamental Study on Masking Considering Biologic Information}

\subsection{Relationship between Heart Rate and Tempo of Music}

In the previous study, we considered creating a comfortable interior sound environment while driving ultra-compact EVs by using masking music including $1 / f$ fluctuation, which provides highly effective relaxation.

However, the music including $1 / f$ fluctuation accounted for only approximately $20 \%$ of all the prepared musical pieces in this experiment. Therefore, we considered that it is difficult to select music including $1 / f$ fluctuation with a high relaxation effect in every driving condition. According to many studies on the relationship between heart rate and tempo of music, when the tempo of music is 1 time, 1.5 times or 2 times the participant's heart rate, the music tends to have a relaxing effect [24-26]. In the experiment, we evaluated the comfort by focusing on the relationship between the heart rate, which is a part of the biologic information and the beats per minute (bpm) of music to select an effective masking music. The tempo of music was calculated as number of beats per minute. The participant's heart rate was measured for 1 min using a heart rate meter, as depicted in Figure 8. We selected 3 music pieces from the 1527 prepared musical pieces from $60 \mathrm{bpm}$ to $238 \mathrm{bpm}$, such that the bpm of the songs was approximately 1 time, 1.5 times and 2 times the heart rate of the participant. There were 16 male participants aged between 21 and 24 years. They were university and graduate school of university students.

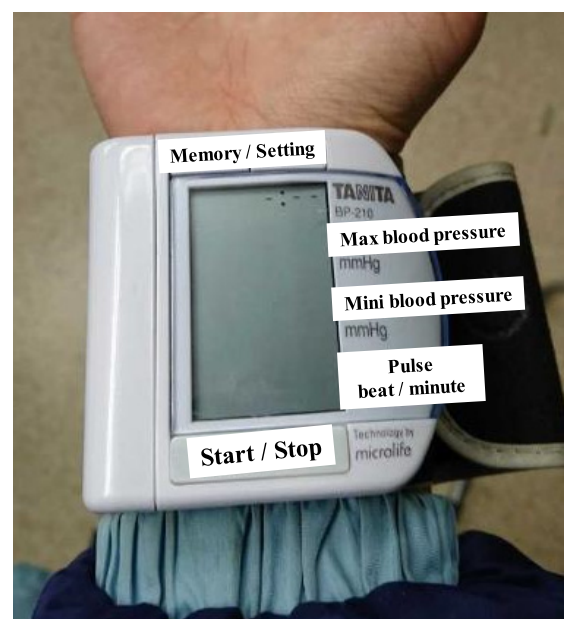

Figure 8. Photograph of heart rate meter.

The ride comfort-based on the relation between the participant's heart rate and tempo of the selected musical piece-was evaluated using the $\alpha$ wave appearance rate, as in the previous experiment in Section 3. The participant wearing the electroencephalograph was seated at rest in front of speakers, which output the assumed road noise and music for masking, as illustrated in Figure 9. The participant's brain wave was measured while he listened to three musical pieces for $3 \mathrm{~min}$ each. The experimental flow is shown in Figure 10.

\subsection{Experiment Result of Brain Wave Appearance Rate under Different Noise Conditions}

Figure 11 depicts the $\alpha$ wave appearance rate of the participants who could relax while listening to music with 1 time, 1.5 times and 2 times the tempo. The results revealed that all the participants were able to be categorized to two different comfort trends. 


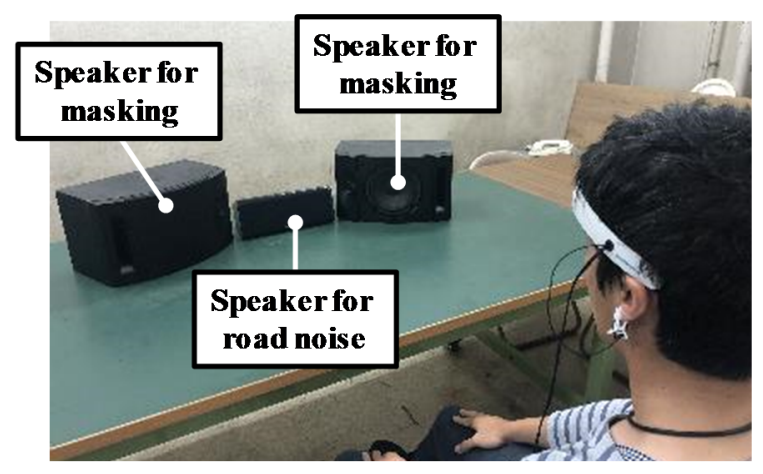

Figure 9. Arrangement of speakers and attached brain wave meter.

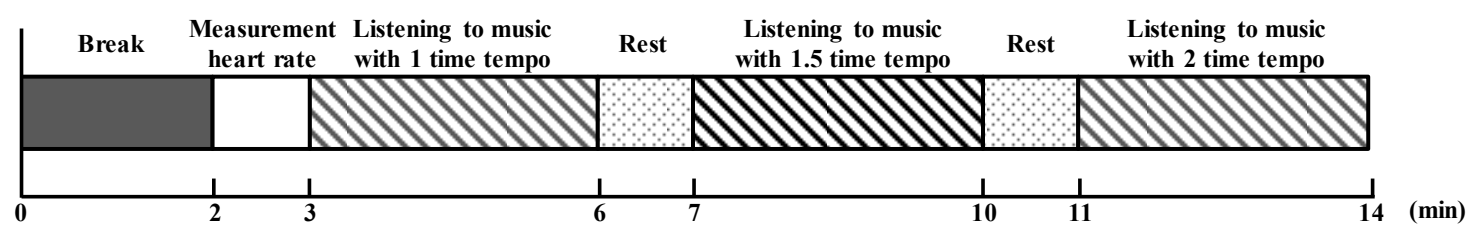

Figure 10. Experimental flow about relationship between heart rate and tempo of music.

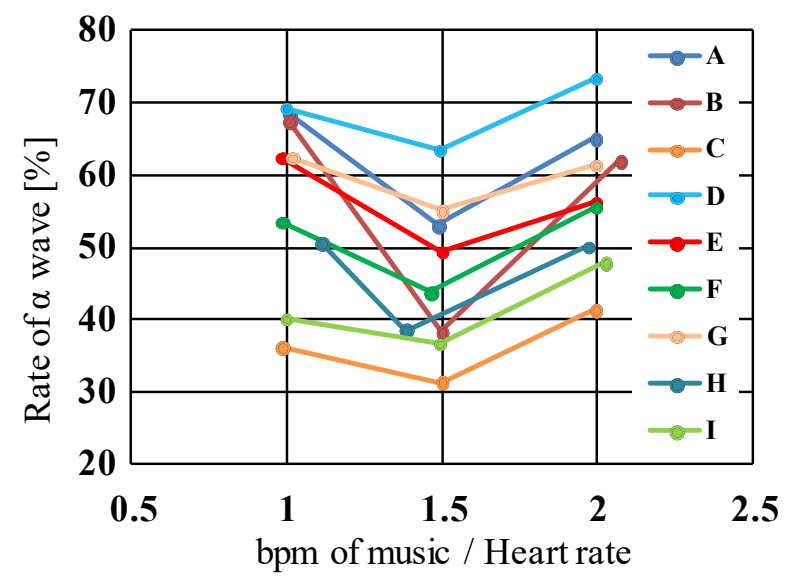

(a)

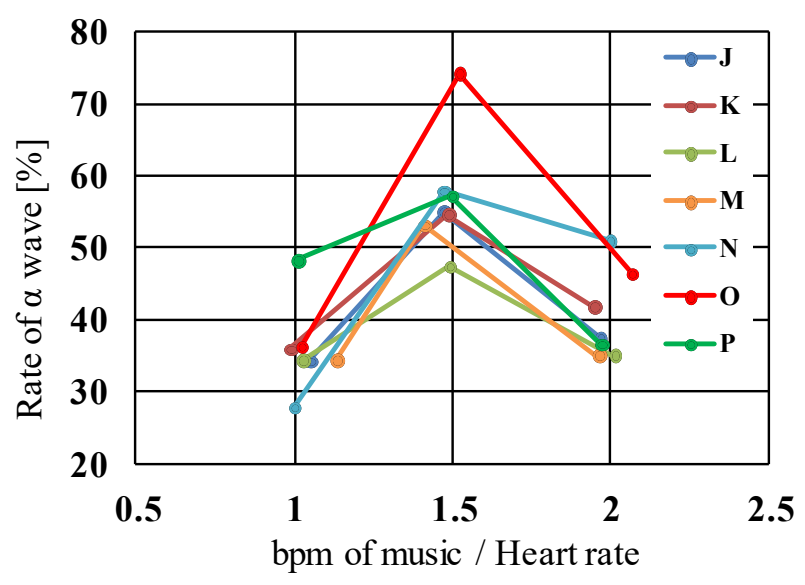

(b)

Figure 11. Experimental result for brain wave appearance rate of different noise condition. (a) $\alpha$ wave appearance rate that participants felt relax when listened to music of 1-time or 2-time tempo; (b) $\alpha$ wave appearance rate that participants felt relax when listened to music of 1.5 -time tempo. 
Thus, it can be concluded that if we can pre-grasp the driver's biologic information of the heart rate and beats per minute of music for masking, the comfort in the interior space can be improved. Therefore, this system can provide a comfortable interior space according to the taste of each drivers.

\section{Masking Experiment Using Driving Simulator}

\subsection{Quantitative Evaluation of Ride Comfort Using Driving Simulator}

According to the results of the previous experiment, we proved the relationship between the heart rate and the $\alpha$ wave appearance rate. We focused on the tempo of music using masking and demonstrated by using the driving simulator that music tempo and heart rate are related to the comfort of the interior space for various drivers.

In this experiment, the participant wore the electroencephalograph and was seated at rest in front of the driving simulator with speakers as in the experiment described in Section 3. The participant's brain wave was measured while listening to three musical pieces for $3 \mathrm{~min}$ each. The participant was given 2 min to prepare for the driving using the driving simulator before measuring the brain wave. Another experiment to select the masking music, measure the heart rate of the participant, and determine the conditions for brain wave measurement was conducted like the experiment described in Section 4.2. In this experiment, there were 12 male participants aged between 21 and 24 years. They were university and graduate school of university students. They had a driving license and more than 3 years of driving experience. The experimental flow is shown in Figure 12.

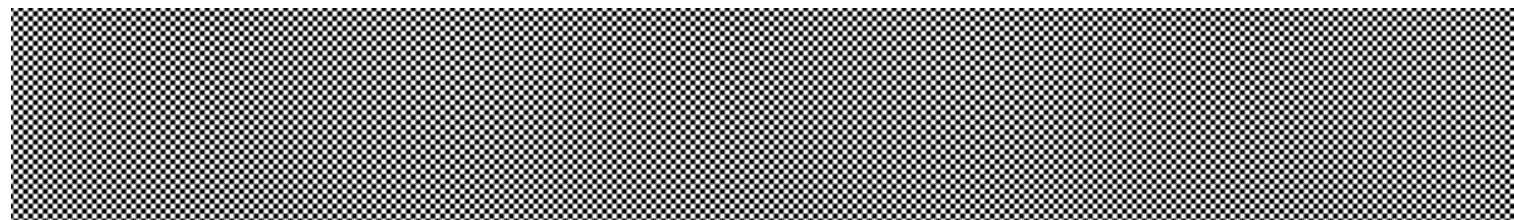

Figure 12. The experimental flow about quantitative evaluation of ride comfort using driving condition.

\subsection{Experiment Result for Brain Wave Appearance Rate of Different Noise Condition during Using Driving} Simulator

Figure 13 depicts the $\alpha$ wave appearance rate of the participants who could relax while listening to music with a tempo of 1 time, 1.5 times and 2 times the participant's heart rate. All participants were found to demonstrate two different comfort trends.

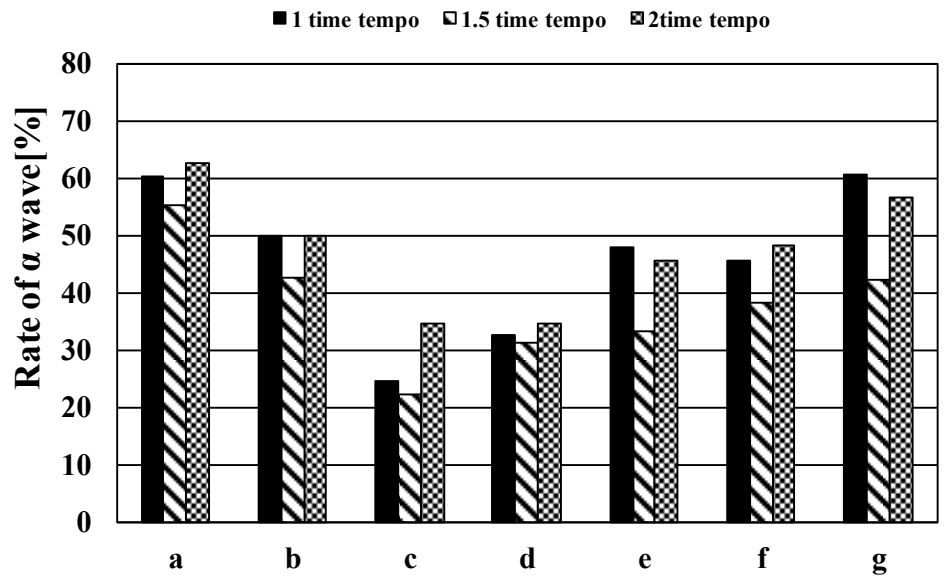

(a)

Figure 13. Cont. 


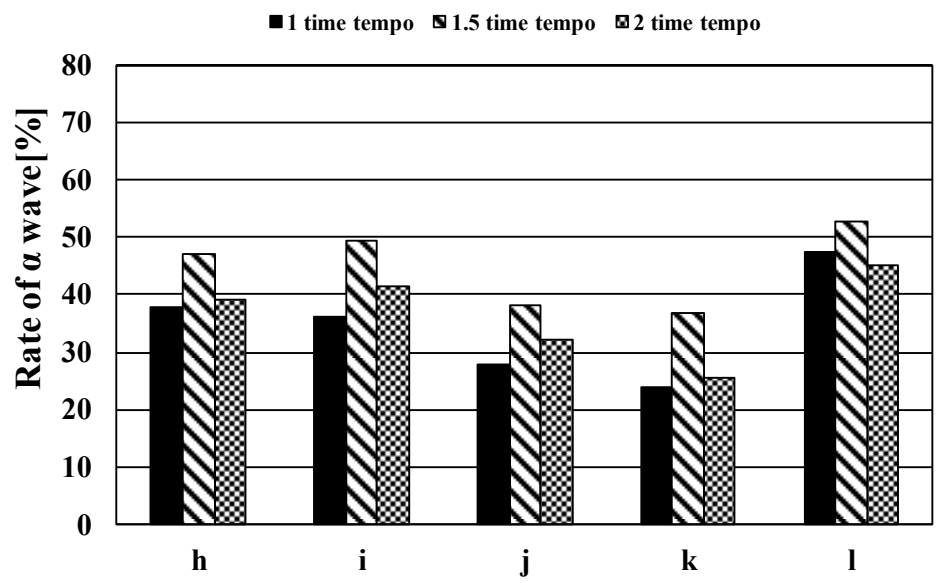

(b)

Figure 13. Experimental results for brain wave appearance rate of different noise condition using driving simulator. (a) $\alpha$ wave appearance rate that participants felt relax when listened to music of 1-time or 2-time tempo; (b) $\alpha$ wave appearance rate that participants felt relax when listened to music of 1.5-time tempo.

Thus, we can conclude that if we can pre-grasp the driver's biologic information of the heart rate and beats per minute of music for masking, then the comfort in the interior space can be improved even when using a driving simulator.

\section{Conclusions}

We conducted this study to quantitatively evaluate the comfort of the interior of an ultra-compact EV by using human biologic information of various drivers.

First, we focused on using the $1 / f$ fluctuation of music for masking the interior noise. The results demonstrated a relaxing effect on the driver. We conducted spectrum analysis on the music pieces that were used for masking and among them, we selected music pieces that included $1 / f$ fluctuation. Second, the participants of the experiment listened to various music pieces under simple driving conditions. Some of the music pieces used by the participants included $1 / f$ fluctuation whereas some others excluded $1 / f$ fluctuation. For all the scenarios, we evaluated the ride comfort of the participants by calculating the $\alpha$ wave appearance rate from brain wave measurement. Furthermore, we investigated the relationship between the heart rate of driver and the tempo of music.

From these results of experiments, the following is a summary of the experimental results in this study.

(1) We could observe an improvement in the driver comfort, when the music that included $1 / f$ fluctuation was used for masking the road noise. The increased level of comfort could be measured by the increased $\alpha$ wave appearance rate in the brain waves of the participants. The participants felt relaxed while listening to the music that included the $1 / f$ fluctuation. The results confirmed that, masking the interior noise in ultra-compact EVs with the music containing $1 / f$ fluctuation can improve the driver comfort.

(2) We evaluated the ride comfort of the participants by focusing on the relationship between the heart rate of driver and the tempo of music used for masking. The $\alpha$ wave appearance rate of the participants while listening to music with a tempo of once, once-and-a-half, and twice their heart rate was observed. The results revealed that, the participants were found to demonstrate two different comfort trends. Further, it was observed that, the comfort of the driver improved with the tempo of music. 
(3) The music pieces that included $1 / f$ fluctuation were approximately $20 \%$ of the total music pieces analyzed for this study. If we pre-grasp the driver's biologic information of the heart rate and beats per minute of music used for masking, the comfort in the interior space can be improved.

From these considerations, if we can pre-grasp the favorite interior sound environment of driver, then the comfort in the interior space can be improved using music for masking according to pattern.

In future research, we aim to create automatic changes in the interior noise conditions by using the driver's biologic information along with the driving conditions, time and environment, even if the driver has a long drive. We also aim to conduct an elaborate study on the topic with larger number of participants.

Author Contributions: T.K., H.N., H.K. and T.N. wrote the study, T.K. and H.N. carried out the experiments; T.K. and H.N. contributed the analyses of the data; H.K. and T.N. conceived ride comfort evaluation by using biologic information for ultra-compact EV. All authors have read and agreed to the published version of the manuscript.

Funding: This research received no external funding.

Acknowledgments: We would like to show our greatest appreciation to Ryosuke Suzuki and Rina Miyao.

Conflicts of Interest: The authors declare no conflict of interest. The founding sponsors had no role in the design of the study; in the collection, analyses or interpretation of data; in the writing of the manuscript and in the decision to publish the results.

\section{References}

1. The Ministry of Land, Infrastructure, Transport and Tourism. The Guideline towards Ultra-Compact Mobility Introduction; The Ministry of Land, Infrastructure, Transport and Tourism: Tokyo, Japan, 2012. (In Japanese)

2. Yukawa, N. Technique of Special Sound Absorbing Sponge. Trans. JSME Jpn. 2009, 112, 426. (In Japanese)

3. Namaizawa, J.; Ishiyama, O.; Yamada, H. Development of road noise prediction method. Jt. Symp. JSME JAS 2001, 1, 143-146. (In Japanese)

4. Yamaguchi, K. Overview of recent issues in sound design for next-generation vehicles due to their quietness. J. Acoust. Soc. 2017, 73, 21-24. (In Japanese)

5. Sanada, A.; Tanaka, N. Active control of sound transmission through a honeycomb panel with feedforward and feedback control. Mech. Eng. J. 2016, 3, 16-00466. [CrossRef]

6. Kato, T.; Suzuki, R.; Miyao, R.; Kato, H.; Narita, T. A Fundamental Consideration of Active Noise Control System by Small Actuator for Ultra-Compact EV. Actuators 2018, 7, 49. [CrossRef]

7. Suzuki, R.; Miyao, R.; Kato, T.; Kato, H.; Narita, T. Improved Interior Acoustic Environment for Ultra-compact EVs (Fundamental Consideration of $1 / f$ Fluctuation Including Music for Evaluation of Ride Comfort). Proc. Sch. Eng. Tokai Univ. Ser. E. 2017, 44, 21-26.

8. Horii, A.; Yamamura, C.; Katsumata, T.; Uchiyama, A. Physiological Response to Unpleasant Sound. J. Int. Soc. Life Inf. Sci. 2004, 22, 536-544. (In Japanese)

9. Tomiyama, K.; Kawamura, A.; Ishida, T.; Takahashi, K.; Akitaya, Y. Development of a Pavement Ride Quality Rating System Using a Driving Simulator and Physiological Information. JSCE Ser. F3 2012, 68, 135-141. (In Japanese)

10. Shu, H.; Song, Y.; Zhou, H. Assessment of Music and Water Sounds for Urban Noise Masking. In Proceedings of the TENCON 2018-2018 IEEE Region 10 Conference, Jeju, Korea, 28-31 October 2018; pp. 1451-1455.

11. Nishiura, T. Unpleasantness reduction of noise based on auditory masking. J Acoust. Soc. 2014, 70, 652-657. (In Japanese)

12. Miyashita, S.; Sunaga, T.; Saito, Y.; Kato, C. A Study of $1 / f$ Fluctuation. Proc. Sch. Media Cent. Hosei Univ. 2008, 21, 85-90. (In Japanese)

13. Nakanishi, R.; Yamamoto, S.; Kiryu, S.; Takeda, S. The Role of Motion Information on Correlations between Music and Emotion (Focusing on Changes between Strain and Relaxation). JSKE 2017, 16, 425-429. (In Japanese)

14. Chinen, Y.; Ikami, K.; Kioka, E. The Image Formed and Feeling Produced by Clothing Colors (From the Relation to the Comfortable Factor Observed in Electroencephalography). JSHE J. Jpn. 2004, 55, 845-851. (In Japanese) 
15. Fukuhara, H. The Relationship between the Brain Response and Wind Band Noise Added to Musical Sound. Noise Control 1989, 13, 224-228. (In Japanese)

16. Tahara, Y.; Inoue, S. Evaluation and Control of Sound Environment Based on Fluctuation Characteristics. Noise Control 2006, 30, 249-257. (In Japanese)

17. Hashimoto, Y.; Noshioka, T.; Umemiya, N.; Okura, R. The Effects of Traffic Noise an EEG Approach. J. Environ. AIJ 2003, 570, 1-6. (In Japanese)

18. Stevenson, D.C.; McKellar, N.R. The Effect of Traffic Noise on Sleep of Young Adults in Their Homes. JASA 1989, 85, 768-771. [CrossRef]

19. Nishijuji, S.; Sato, M.; Maino, D.; Tanaka, S. Effect of Acoustic Environment on EEG during Mental Task. Life Support 2010, 22, 10-18. (In Japanese)

20. Ishizuka, K.; Kato, T.; Kato, H.; Narita, T.; Kojima, A.; Moriyama, H. Active Noise Control for Ultra-Compact Vehicle Using Giant Magnetostrictive Actuator (Comfort Evaluation of the Vehicle Interior Noise by EEG). Trans. JSME Jpn. 2017, 25, 88-93. (In Japanese)

21. Kato, T.; Suzuki, R.; Narita, T.; Kato, H.; Yamamoto, Y. Basic Study on Active Noise Control for Considering Characteristics of Vibration of Plate by Giant Magnetostrictive Actuator. IJAEM 2016, 52, 153-160. [CrossRef]

22. Musya, T. $1 / f$ Fluctuations and Pleasant Sensation. ASJ J. 1994, 50, 485-488. (In Japanese)

23. Doi, S.; Otsuka, T.; Takahashi, H. Experimental Investigation on Lighting Control with $1 / f$ Fluctuation. Trans. IEEJ C 1997, 117, 409-415.

24. Matsuda, K.; Ichikawa, M.; Tachibana, K. The Influence of Heart Rate on Time Perception during Music Listening. Trans. JSKE 2015, 14, 215-222.

25. Hotta, H.; Awamura, K.; Inoue, T. Heart Rate Variability during Music Listening at the Same Tempo as Subject's Heart Rate. J. Educ. Psychol 2007, 33, 1-8.

26. Takenaka, M.; Okai, S.; Kohara, Y.; Inoue, K. A Psychophysiological Study on Biological Reaction Using Heart Rate Rhythm. J. Clin. Psychol. 2005, 31, 1-13.

(C) 2020 by the authors. Licensee MDPI, Basel, Switzerland. This article is an open access article distributed under the terms and conditions of the Creative Commons Attribution (CC BY) license (http://creativecommons.org/licenses/by/4.0/). 